\title{
Application of digital particle image velocimetry to insect aerodynamics: measurement of the leading-edge vortex and near wake of a Hawkmoth.
}

RICHARD J. BOMPHREY, NiCHOLAS J. LAWSON*, GRAHAM K. TAYLOR \& ADRIAN L. R. THOMAS

Department of Zoology, University of Oxford, South Parks Road, Oxford OX1 3PS, U.K.,

*School of Engineering, Cranfield University, Cranfield, MK43 0AL, U.K.

Correspondence to R. J. B. (e-mail: Richard.Bomphrey@zoo.ox.ac.uk).

\section{Abstract}

Some insects use leading-edge vortices to generate high lift forces, as has been inferred from qualitative smoke visualisations of the flow around their wings. Here we present the first Digital Particle Image Velocimetry (DPIV) data and quantitative analysis of an insect's leading-edge vortex and near wake at two flight speeds. This allows us to describe objectively two-dimensional slices through the flow field of a tethered Tobacco Hawkmoth (Manduca sexta). The near-field vortex wake appears to braodly resemble elliptical vortex loops. The presence of a leading-edge vortex towards the end of the downstroke is found to coincide with peak upwards force production measured by a six component force-moment balance. The topology of Manduca's leading-edge vortex differs from that previously described in that late in the downstroke, the structure extends continuously from wingtip across the thorax to the other wingtip. 


\section{List of symbols}

F $\quad$ Time averaged locomotor force

$\rho \quad$ Air density

$\Gamma \quad$ Circulation

A Vortex loop area

$T \quad$ Wingbeat period

$d \quad$ Diameter of vortex core

$\omega_{t} \quad$ Mean tangential velocity at edge of vortex core

$V_{\infty} \quad$ Freestream velocity

\section{Key words:}

Flow visualisation wake Leading-edge vortex Manduca sexta DPIV unsteady aerodynamics flapping wing aerofoil.

\section{1}

\section{Introduction}

The aerodynamic mechanisms that insects use have received a great deal of experimental and theoretical attention as they generate greater than normal lift coefficients (Azuma and Watanabe 1988; Birch and Dickinson 2001; Bomphrey et al. 2002; Dickinson et al. 1999; Ellington et al. 1996; Somps and Luttges 1985; Srygley and Thomas 2002; Willmott et al. 1997; Zbikowski 2002). To date, the aerodynamic mechanisms used by insects have been inferred by eye from qualitative flow visualisations. Smoke visualisations with tethered hawkmoths (Ellington et al. 1996) have been interpreted as showing a conical leading-edge vortex spiralling spanwise 
from a point fixed on the proximal surface of the wings (a focus). Experiments with flapping mechanical models have shown qualitatively the same flow structures (Ellington et al. 1996; Maxworthy 1979; Van den Berg and Ellington 1997), with significant spanwise flow extending vorticity into the tip vortices to stabilise the flow structure. More recent work (Birch and Dickinson 2001) indicates that spanwise flow is insignificant at the lower Reynolds numbers associated with smaller insects ( $R e$ 100). Recent smoke visualisations with free-flying butterflies (Vanessa atalanta) (Srygley and Thomas 2002) reveal another class of leading-edge vortex, in which the structure is continuous across the insect's centreline, with no surface bound focus (i.e. with a free-slip critical point over the centreline).

A major difficulty in interpreting smoke visualisations is that they record a summed time history of a flow, rather than the instantaneous flow field. However, new insights into the flow are possible if instantaneous data are recorded from the flow using a technique such as Particle Image Velocimetry (PIV) (Adrian 1991). In the following Digital PIV (DPIV) is used to measure the flow around the wings and in the near wake of a Tobacco Hawkmoth (Manduca sexta L.).

\section{2}

\section{Materials and Methods}

Several individuals of the Tobacco Hawkmoth Manduca sexta L. were obtained from a captive bred population, and selected for physical characteristics indicative of health (good wing condition, strong free-flight ability, etc.). Data presented here were collected over 9 consecutive bouts of flight from an individual weighing $1.52 \mathrm{~g}$, with a wing length of $52 \mathrm{~mm}$ and a thorax width of $8 \mathrm{~mm}$. From image analysis, the body angle was found to be elevated by $28^{\circ}$ from horizontal. The insect was flown for DPIV measurements in a low-speed, low-turbulence wind tunnel $(1.0 \times 0.5 \times 0.5 \mathrm{~m}$ working section) at two flight speeds where the freestream was set at $V_{\infty}=1.2 \mathrm{~ms}^{-1}$ and $3.5 \mathrm{~ms}^{-1}$. The insect was rigidly tethered (using cyanoacrylate adhesive applied to the underside of the thorax) to a 6-component strain gauge force-moment balance (FFA I666; $10 \mathrm{kHz}$ sampling rate) connected to a Macintosh computer (with MacLab 
hardware and Chart v.3.6/s) and synchronised with the DPIV system. The balance output was converted to force-moment units in all channels in Matlab using a static calibration analysed as a third order general linear model (GLM) with all significant second order interactions ( $P=0.05$; Taylor and Thomas, in prep.).

A DPIV system was set-up to generate a fixed vertical light sheet that was projected into the centre of the working section of the wind tunnel from the downstream tunnel exit (Fig. 1). The light sheet was generated from a New Wave Gemini Nd:YAG pulsed laser (120mJ per $5 \mathrm{~ns}$ pulse) and a combination of a plano-concave and planocylindrical lenses with focal lengths of $-50 \mathrm{~mm}$ and $75 \mathrm{~mm}$ respectively. This resulted in a $0.4 \mathrm{~mm}$ thick expanding light sheet that provided an imaging area up to $100 \mathrm{~mm} \times$ $100 \mathrm{~mm}$. The force balance could be traversed across the tunnel with the moth in situ which allowed planes to be measured from the moth at either midspan or abdomen (sagittal plane) positions. Seeding was supplied from a JEM Hydrosonic Seeder (Lancelyn Theatre Supplies, Oxford, U.K) placed at the tunnel intake with 'long lasting' smoke fluid to produce seeding with a diameter $<10 \mu \mathrm{m}$. DPIV images were recorded using a Micro-Nikor 105mm focal length lens and Kodak ES1.0 CCD synchronised to the laser through a Dantec Flowmap 500 acquisition box and Flowmanager software. Laser pulse separations of between $70-200 \mu$ s were used depending on the freestream velocity. Around 70 pairs of images were taken for each flight session. At each windspeed, two flights were recorded with the light sheet in the sagittal plane of the animal, and two flights with the light sheet in a para-sagittal plane (midspan on the wing nearest the camera). From these flights a representative sample of DPIV images was selected to compile a time-resolved history of the flow field. Ideally a full time-resolved set of vector maps would be desirable, but the $15 \mathrm{~Hz}$ frame rate of the ES1.0 was insufficient to do this for the $19 \mathrm{~Hz}$ moth wingbeat frequency.

DPIV images were processed using TSI UltraPIV Insight software incorporating the Hart Algorithm (Hart 2000). A primary correlation window of $32 \times 32$ pixels was selected with a sub-correlation window of $16 \times 16$ pixels and a search radius of $8 \times 8$ pixels. A bilinear peak search was also selected. This typically yielded $121 \times 121$ vectors per DPIV image and corresponded to a spatial resolution of $0.75 \mathrm{~mm} \times$ $0.75 \mathrm{~mm}$ over a $92 \mathrm{~mm} \times 92 \mathrm{~mm}$ imaging area. Vector and vorticity fields were displayed using Tecplot v.8.0 and streamlines were also added using the pull-down 
menu option. These 'streamlines' are based solely on the 2-dimensional slice of data through a 3-dimensional wake and are thus projections onto a plane rather than classical streamlines. The flow is also unsteady, and for both these reasons, it is unlikely that any particle would ever travel along these lines. The streamlines presented here are, however, still able to define flow structures such as vortices. From error analysis outlined previously (Lawson and Davidson 2001), it is estimated that the error in the DPIV data is in the range $2.2 \%$ - 5.5\% of full scale measurement.

\section{3}

\section{Analysis}

Because the primary aim of this study was to visualise the fluid motion in the immediate vicinity of the wing, the camera's field of view was too small to visualise the far wake except at the lower speed. At this speed $\left(V_{\infty}=1.2 \mathrm{~ms}^{-1}\right)$, we were able to infer the aerodynamic forces on the wings by measuring the circulation of the trailing, or wingtip vortices convected in the wake using cross sections in the plane of the laser. This inference is justified by Kelvin's theorem of equivalence in the absolute magnitude of bound and shed circulation during the impulsive starting of an aerofoil or hydrofoil under inviscid conditions. Since the wings are held close together dorsally at the end of each complete wingbeat, and some insects have been shown at relatively slow speeds such as these to produce structures approximating vortex rings, we calculate locomotor forces from vortex loops (which need not be round) using these assumptions as a starting point. The loop created by each wingbeat cycle is the combination of wingtip kinematics and the effect of the freestream on the convecting trailing vortices. Assuming that the conditions are inviscid, and that all the momentum added to the fluid by the moth is contained within vortices in the wake, the time averaged locomotor force $F$ can be calculated as follows:

$F=\rho \Gamma A / T$

where $\rho$ is the air density $\left(1.225 \mathrm{~kg} \cdot \mathrm{m}^{-3}\right), \Gamma$ is the circulation (the line integral of the tangential velocity component about a curve enclosing the vortex core; (Batchelor 
1967)), again, each of these velocities is a projection of the total possible velocity vector onto the plane of interrogation defined by the laser. $A$ is the area enclosed by the vortex loop, and $T$ is the time over which the force is generated (i.e. one wingbeat period). $\Gamma$ was calculated by the equation:

$\Gamma=\pi \cdot d \cdot \omega_{t}$

where $d$ is the diameter of the vortex core and $\omega_{\mathrm{t}}$ is the mean tangential velocity at the edge of the vortex core. The edge of the vortex core was defined as the point at which maximum and minimum velocities occurred on a velocity profile through the core (measured as a mean of the peak velocities in horizontal and vertical crosssections through the same vortex core on each image), and it was assumed that there was no significant degradation of the vorticity during the small convection from wing tip to the point of measurement. The area of the loop was approximated and calculated as for an ellipse with the major axis (core centre to core centre) measured from the calibrated DPIV images, and the minor axis taken as the length of two wings plus the thorax width. This approximation was used in all calculations, as the field of view was only big enough to contain a complete loop as soon as it had shed, owing to convection of the earliest shed sections of the vortex with the freestream. This is justified in that the minor axis is defined by the individual's geometry, and the major axis is dependent on wing beat frequency and the freestream velocity, both of which remained constant throughout each of the separate experimental periods. The angle of the loop to horizontal ( $x$-axis of images) and the jet angle were measured using Tecplot.

\section{4}

\section{Results and Discussion}

An insect wing stroke cycle consists of four phases. Conventionally speaking it begins with a rotational phase at the top of the upstroke and beginning of the downstroke called 'pronation'. There follows a largely translational downstroke 
phase, followed by a second rotation called 'supination', and finally the translatory upstroke. The flow field induced by the moth towards the end of the downstroke at the commencement of supination is shown in Fig. 2, with the $V_{\infty}=3.5 \mathrm{~ms}^{-1}$ freestream velocity subtracted. At this point in the wingbeat cycle, the camera looks directly down the length of the near/left wing, as it begins to rotate in preparation for the upstroke. The flow halfway out along the wings (Fig. 2a-c) is separated at the leading edge, forming a leading-edge vortex. Owing to the controversy over vortex identification methods, it has become common practice to use a combination of two or more criteria to reduce the likelihood of misidentification (Banks and Singer 1995). One criterion is that of streamlines spiralling into a stable focus (Robinson 1991); another is that of a vorticity maximum (Lugt 1985). On the downstroke of Manduca, the streamlines converge in a stable focus above the wing. This coincides with a vorticity maximum, demonstrating the presence of a leading-edge vortex above the wing. The leading-edge vortex coincides with peak upward force production, and is equivalent to 2.2 times bodyweight (Fig. 3). Inertial forces due to acceleration and deceleration of the wings and their associated added mass have not been removed from the force traces, but since the wings decelerate in preparation for stroke reversal, these inertial forces would then be acting downwards, implying an aerodynamic contribution even greater than 2.2 times bodyweight.

The leading-edge vortex was clearly observed at $V_{\infty}=3.5 \mathrm{~ms}^{-1}$, and was recorded during a sequence in which the moth was supporting $79 \%$ of its bodyweight (averaged over 10 wingbeats). There is similar flow separation at $V_{\infty}=1.2 \mathrm{~ms}^{-1}$. The frame of reference is crucial if straightforward subtraction of the freestream is to reveal a focus. Over the thorax, subtraction of the full freestream velocity at $10^{\circ}$ downwards deflection revealed a focus, which coincided with the point of peak vorticity (Fig. 2d). For Fig. 2d, some areas were blanked on account of glare from the thorax and abdomen. No rotational transformation of the vector map was introduced but there is still a correlation of converging streamlines and a vorticity maximum.

Velocity profiles of cross sections through the loops shed into the wake are similar to sections through ideal vortex rings and their cores (Fig. 3) derived from potential theory, though there are minor differences. The top three pairs of graphs in Fig. 3 
show the close correlation between theoretical velocity profiles across vortex rings (a), cores (b) and axes (c), and those obtained through experimentation with Manduca. (d) shows a similar pair of vertical and horizontal profiles through the leading-edge vortex. The width of a core is considered to be the portion of the graph which approximates to a straight line indicating solid body rotation - that portion of the graph between the maximum and minimum values. In the ideal case, measured velocities in Fig. 3b and 3c both should decay to zero at the edges of the graphs but do not, due to the overall downward convection of the vortex loop and core. The leadingedge vortex at midspan is approximately elliptical in cross-section with the major axis directed chordwise. This can be seen from the shape of the streamlines and vorticity in Fig. 2, and also measured from the vertical and horizontal $u$ and $v$ component velocity profiles (Fig. 3d). At the instant shown in Fig. 2, its core is $4 \mathrm{~mm} \times 6 \mathrm{~mm}$ (Fig. 3d, measured from those portions of the cross-sectional profiles which can be approximated to solid body rotation of the vortex core.). This is marginally smaller at this midspan position than the freely convecting earliest shed tip vortex cores seen at $V_{\infty}=1.2 \mathrm{~ms}^{-1}$, which were typically in the order of $8 \mathrm{~mm}$ diameter ( $\mathrm{n}=15$ image pair correlations, standard deviation $=2.2$ ). Figure $3 \mathrm{c}$ shows an example of a vortex core cross-section.

Figure 3e shows the vertical (positive upwards) and horizontal (positive forwards) force measurements of the same wingbeat (i.e. the wingbeat from which the presented leading-edge vortex images were captured). 'Relative force' is force relative to body mass where a vertical (positive) value of 1 means the animal was exactly supporting its weight, including both aerodynamic lift, and inertial forces. The dashed line indicates the instant the laser fired coincides with the peak in force generation. The insert shows the vertical force trace for the surrounding ten wingbeats. The main graph corresponds to the yellow section of the insert, and is not exceptional. The wake behind the wings consists of a curved trailing region of higher velocity flow (green/blue Fig. 2c). These velocity values are a 2-dimensional projection of the real 3-dimensional vectors using velocity components in the plane of interrogation. At the uneven lower boundary of this region is a vortex sheet shed from, and following the path of the trailing edge of the wing. This vortex sheet represents a difference in flow velocity above and below the trailing edge. Once this difference in velocity reaches a critical value, Kelvin-Helmholtz instability will cause the vortex sheet to roll up into a 
series of discrete transverse vortices. Support for Kelvin-Helmholtz roll up can be seen in Fig. 2 (a and c) although the uneven lower boundary could be due to uneven wing flapping, which is impossible to determine without detailed kinematics.

If we attempt to describe the dominant structures in the wake of Manduca in their simplest form, they consist of a series of non-planar (i.e. curved) approximately elliptical vortex loops. The earliest and latest portions of the loop are approximately level horizontally, but the plane of the loop defined by the vortex core is curved downwards between these areas. The structures are certainly not classical axisymmetric vortex rings in that the jet angle is not in line with the axis, and the word 'ring' implies a circular shape. They are only approximately elliptical, however, because of the ill-defined shedding of the bound vortex after the end of the downstroke. The loops are initially formed along the path traced by the wingtips, but the earlier formed sections convect downwards as the wings progress through the stroke. Fig. 4 is a composite sequence highlighting the convection of the early sections of the vortex loop - with shed vorticity shown in green and yellow - from its creation with wings held aloft and close together (Fig. 4a), to its location at the point after the wings begin their upstroke (Fig. 4d).

With the trailing edges of the wings still held close together dorsally, the leading edges begin to pull apart, creating counter-rotating vorticity (shown as blue and green areas) around the wings and in the near wake (Fig. 4a). Fig. 4b shows that part of the loop which was earliest to form (vorticity shown in green) convecting backwards and downwards in the wake as the moth induced flow grows and becomes stronger. Rollup of the early trailing vortex (or wingtip vortex) is complete in Fig. 4c as the loop takes shape and the yellow-green patch of vorticity is shed and becomes distict from the wings. This first part of the loop to be formed by the wingtips convects downwards and backwards until it is horizontally level with the vorticity still bound onto the wing (Fig. 4d). The final structure therefore incorporates both the trace of the wingtip motion and the history of convection of the earliest-shed sections of the wake. The moth induced flow is now dominated by a downwards and backwards pointing flow, indicated by the largest red vectors. Horizontal cross-stream vorticity (normal to the plane of the paper/field of view) is shed in the form of a shear layer behind the trailing edges. Calculations based on the geometry of the gross loop structure used 
only the major vortical structures visualised in the wake. Fig. 4 also shows some minor patches of vorticity which are shed into the wake, but not accounted for in these calculations.

A cartoon of the complete loop is shown in Fig. 5 highlighting the main wake elements with their associated rotations, the major deflection of the freestream, and also the minor filaments (cross-stream red lines) which form in the curved plane of the vortex sheet after the shear layer has deteriorated. The width of the ellipse can be assumed to be the tip-to-tip span of the moth; the length of the ellipse is a combination of factors including wingbeat frequency and freestream velocity (1.8 times longer than it's width at $V_{\infty}=1.2 \mathrm{~ms}^{-1}$ ). The final shape of the loop is a horizontal, elliptical torus, curved downwards in the middle, with a jet running approximately down through the loop's main axis. The horizontal stopping vortex normal to the freestream (the last section of the loop to be shed into the wake) is shed well into the upstroke, and is more diffuse than other parts of the loop. This is perhaps as a result of the interference of subsequent upstroke wake (Spedding 1987), but it is unlikely to be as clearly defined as the earliest shed trailing wingtip vortex. This is because the wings do not approach each other closely ventrally as they do dorsally. Vorticity is shed continuously from the trailing edges, and is predominantly anticlockwise (in the same reference frame as Fig. 2 and Fig. 4), indicating a complementary increase in the circulation around the wings during the downstroke, most likely linked with the growth of any leading-edge vortex that may form.

Kelvin's theorem that the absolute magnitude of bound and shed vorticity is equivalent allows us to use the circulation of the wake vortices to infer the propulsive forces that arise from circulation around the wings. The DPIV data suggest that the forces produced by the vortex loops can account for $85 \%$ of the measured resultant force averaged through the stroke (standard deviation=26\%, $n=15$ image pairs). The jet through the downstroke loop is directed approximately $18^{\circ}$ back from the loop's axis, so generates lift and thrust in a 3:1 ratio.

Clapping, or near clapping, of the wings (Weis-Fogh 1973) also produces a discrete vortex loop (Fig. 6): an infrequent feature of the flow that will contribute additional propulsive force. Using the same approach to calculate the thrust generated by the 
clap loops suggests that they produced 2.5 times that generated by the downstroke loops (mean thrust from downstroke loops $=1.25 \mathrm{mN}$, std $=0.48 \mathrm{mN}, n=15$ image pairs; mean thrust from clap loops $=3.12 \mathrm{mN}$, standard deviation $=0.39 \mathrm{mN}, n=3$ image pairs). Not all wingbeats concluded with the formation of a clap loop, but these calculations indicate that claps can generate considerable thrust to supplement that generated by the downstroke loops. Clap loop jet angles ranged widely from $20.9^{\circ}$ to $40.3^{\circ}$, consistent with the hypothesis that claps may be used for pitch control (Taylor 2001).

Previous studies (Maxworthy 1979; Van den Berg and Ellington 1997) have suggested that leading-edge vortices used by insects are analogous in some respects to those found on delta wing aircraft. In particular, it has been suggested that the leading-edge vortex may be stabilised by a strong spanwise flow toward the wing tip. Van den Berg and Ellington (1997) deduced that the spanwise flow on their robotic hawkmoth was of the same magnitude as the swirl (speed of rotation around the vortex axis) using qualitative techniques. In 2D DPIV this strong out of plane component will create a perspective error which is dependent on the position in the object plane as a percentage of the object distance (Reeves and Lawson 2003; Soloff et al. 1997). In this case, if the out of plane component is the same order as the inplane component, by using a lens with a focal length of $105 \mathrm{~mm}$ and an object plane of around $100 \mathrm{~mm} \times 100 \mathrm{~mm}$, the perspective error has a maximum of $5 \%$ at the edge of the field. This reduces to zero in the centre of the field and accounts for the range of error of $2.2 \%-5.5 \%$ in the error analysis. The common approach to remove this error is to use a stereoscopic DPIV arrangement (Prasad 2000) which is the subject of further work.

\section{5}

\section{Conclusions}

DPIV and force balance data have been presented from a tethered Tobacco Hawkmoth (Manduca sexta). The DPIV data has allowed quantitative analysis of the 
insect's wing flow structures and wake. These DPIV results have shown the presence of a significant leading-edge vortex towards the end of the downstroke. This flow feature confirms results from previous flow visualisation (Ellington et al. 1996). Direct comparisons of the DPIV results to simultaneous six component force data have also shown the peak upwards force to coincide with the presence of the leadingedge vortex at the end of the downstroke. Lift calculations from the DPIV data using circulation theory match to within $15 \%$ of the force balance data. The discrepancies are in part attributed to the 3D nature of the flow and the corresponding measurement errors generated in this case with a 2D DPIV system. The gross structure of the near wake resembles vortex loops, particularly at the slower of two flight speeds $\left(1.2 \mathrm{~ms}^{-1}\right)$. The structure of the LEV at both flight speeds resembles that previously described only at the midwing position, but is shown to continue over the thorax and centreline of the aninal, and be continuous with both trailing vortices from the wings during late downstroke.

\section{Acknowledgements}

Work funded by BBSRC grant. G.K.T. is a Royal Society University Research Fellow and Weir Junior Research Fellow at University College, Oxford.

\section{References}

Adrian RJ (1991) Particle-imaging techniques for experimental fluid mechanics. Ann. Rev. Fluid Mech. 23:261-304

Azuma A, Watanabe T (1988) Flight performance of a dragonfly. J. Exp. Biol. 137:221-252

Banks DC, Singer BA (1995) A Predictor-Corrector Technique for Visualizing Unsteady-Flow. IEEE Trans. Vis. Comput. Graph. 1:151-163

Batchelor GK. (1967) An introduction to fluid dynamics. Cambridge: Cambridge University Press

Birch JM, Dickinson MH (2001) Spanwise flow and the attachment of the leadingedge vortex on insect wings. Nature 412:729-733 
Bomphrey RJ, Srygley RB, Taylor GK, Nudds RL, Thomas ALR (2002) Visualising of the flow around insect wings. Physics of Fluids. 14(9):S4

Dickinson MH, Lehmann F-O, Sane SP (1999) Wing rotation and the aerodynamic basis of insect flight. Science 284:1954-1960

Ellington CP, vandenBerg C, Willmott AP, Thomas ALR (1996) Leading-edge vortices in insect flight. Nature 384:626-630

Hart DP (2000) PIV error correction. Exp. Fluids 29:13-22

Lawson NJ, Davidson MR (2001) Self-sustained oscillation of a submerged jet in a thin rectangular cavity. Journal of Fluids and Structures 15:59-81

Lugt HJ (1985) Vortex Flow and Maximum-Principles. Am. J. Phys. 53:649-653

Maxworthy T (1979) Experiments on the Weis-Fogh mechanism of lift generation by insects in hovering flight. Part 1. Dynamics of the 'fling'. J Fluid Mech. 93:4763

Prasad AK (2000) Stereoscopic particle image velocimetry. Exp. Fluids 29:103-116

Reeves M, Lawson NJ (accepted Sept 2003) Evaluation and correction of perspective errors in endoscopic PIV. Exp Fluids

Robinson SK (1991) Coherent Motions in the Turbulent Boundary-Layer. Annu. Rev. Fluid Mech. 23:601-639

Soloff SM, Adrian RJ, Liu ZC (1997) Distortion compensation for generalized stereoscopic particle image velocimetry. Measurement Science \& Technology 8:1441-1454

Somps C, Luttges M (1985) Dragonfly Flight - Novel Uses of Unsteady Separated Flows. Science 228:1326-1329

Spedding GR (1987) The wake of a kestrel (Falco tinnunculus) in flapping flight. J. Exp. Biol. 127:59-78

Srygley RB, Thomas ALR (2002) Unconventional lift-generating mechanisms in freeflying butterflies. Nature 420:660-664

Taylor GK (2001) Mechanics and aerodynamics of insect flight control. Biological Reviews 76:449-471

Van den Berg C, Ellington CP (1997) The three-dimensional leading-edge vortex of a 'hovering' model hawkmoth. Phil. Trans. R. Soc. Lond. B 352:329-340

Weis-Fogh T (1973) Quick estimates of flight fitness in hovering animals, including novel mechanisms for lift production. J Exp Biol 59:169-230

Willmott AP, Ellington CP, Thomas ALR (1997) Flow visualization and unsteady aerodynamics in the flight of the hawkmoth, Manduca sexta. Phil. Trans. R. Soc. Lond. B 352:303-316

Zbikowski R (2002) On aerodynamic modelling of an insect-like flapping wing in hover for micro-air vehicles. Phil. Trans. R. Soc. Lond. A 360:273-290

\section{Figure legends}

Figure 1. Plan view schematic diagram of the experimental apparatus. 
Figure 2. (a) and (b - magnified) show vorticity plots of the flow around the wings of Manduca and in its near wake with the light sheet aligned with the midwing position. (c) shows a contour plot of the squared, in-plane velocity component, with velocity vectors overlaid to highlight the shape of the near wake. (d) shows a similar image to (b) with the light sheet at the centreline $\left(V_{\infty}=3.5 \mathrm{~ms}^{-1}\right)$.

Figure 3. $(\mathrm{a}-\mathrm{c})$ Velocity profiles through ideal and measured vortex loops $\left(V_{\infty}=1.2 \mathrm{~ms}^{-1}\right)$. (d)

Velocity profiles through the leading-edge vortex $\left(V_{\infty}=3.5 \mathrm{~ms}^{-1}\right)$. e) Typical force measurements $\left(V_{\infty}=\right.$ $\left.3.5 \mathrm{~ms}^{-1}\right)$.

Figure 4. DPIV composite sequence to show the production of a vortex loop $\left(V_{\infty}=1.2 \mathrm{~ms}^{-1}\right.$ : light sheet on centreline: blank areas indicate data loss through glare, occlusion or insufficient contrast between particles and background, i.e. the moth body). (a) represents the initial pulling apart of the wings, (d) represents an instant shortly after supination at the beginning of the upstroke.

Figure 5. Production of a vortex loop with cross-sections of the loop core $\left(V_{\infty}=3.5 \mathrm{~ms}^{-1}\right)$.

Figure 6. Jet formation after a clap $\left(V_{\infty}=1.2 \mathrm{~ms}^{-1}\right.$ : light sheet on centreline). 
Richard J. Bomphrey; Nicholas J. Lawson; Graham K. Taylor; Adrian L. R. Thomas. Application of digital particle image velocimetry to insect aerodynamics: measurement of the leading-edge vortex and near wake of a Hawkmoth. Experiments in fluids, 2006, vol. 40, no4, pp546-554.

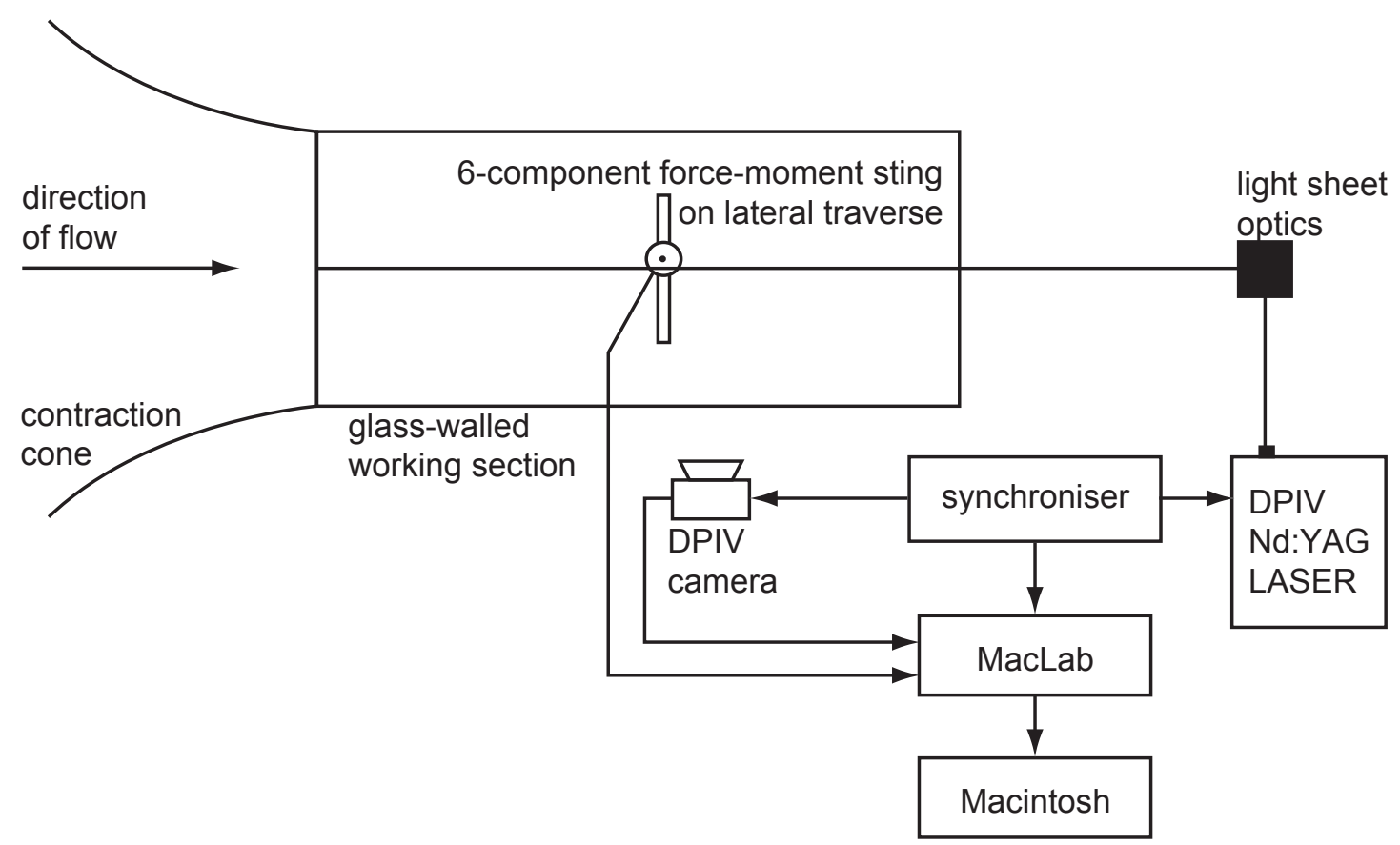


Figure 2 Bomphrey et al.
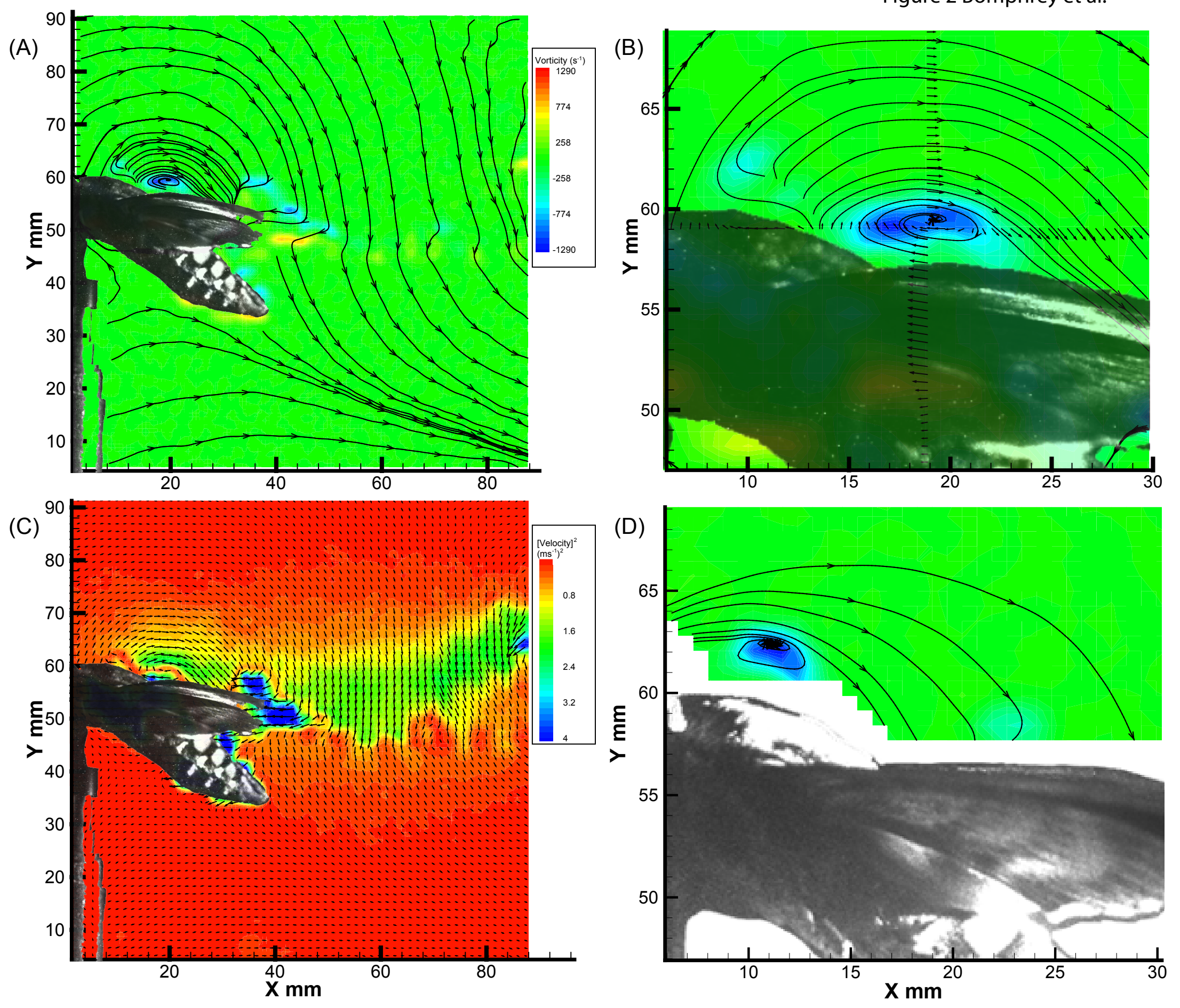
Bomphrey et al. Fig 3 Velocity profiles through vortex loops

Ideal (theoretical)

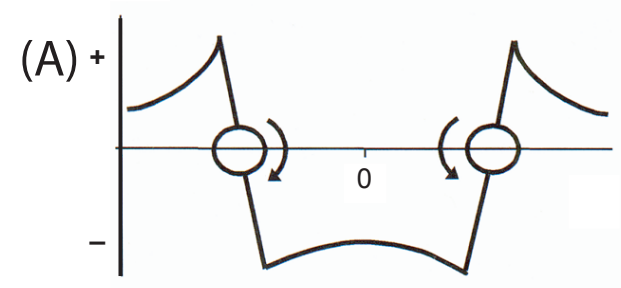

$(\bigcirc$

(B)

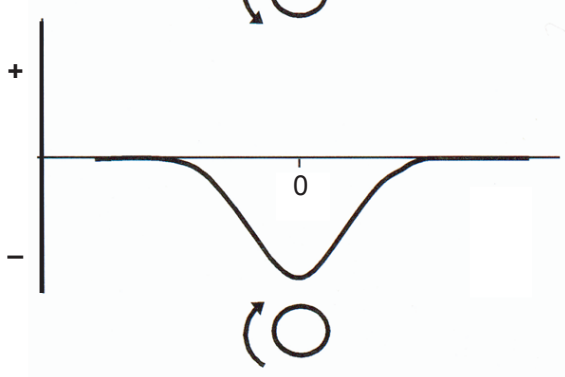

(C)

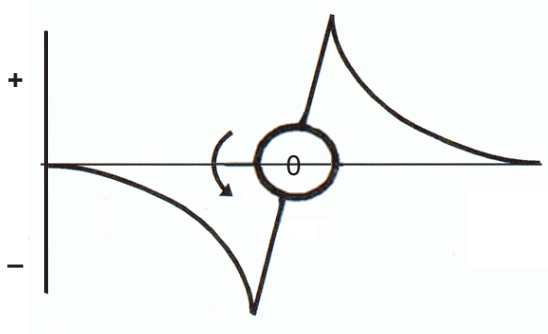

Measured (experimental)
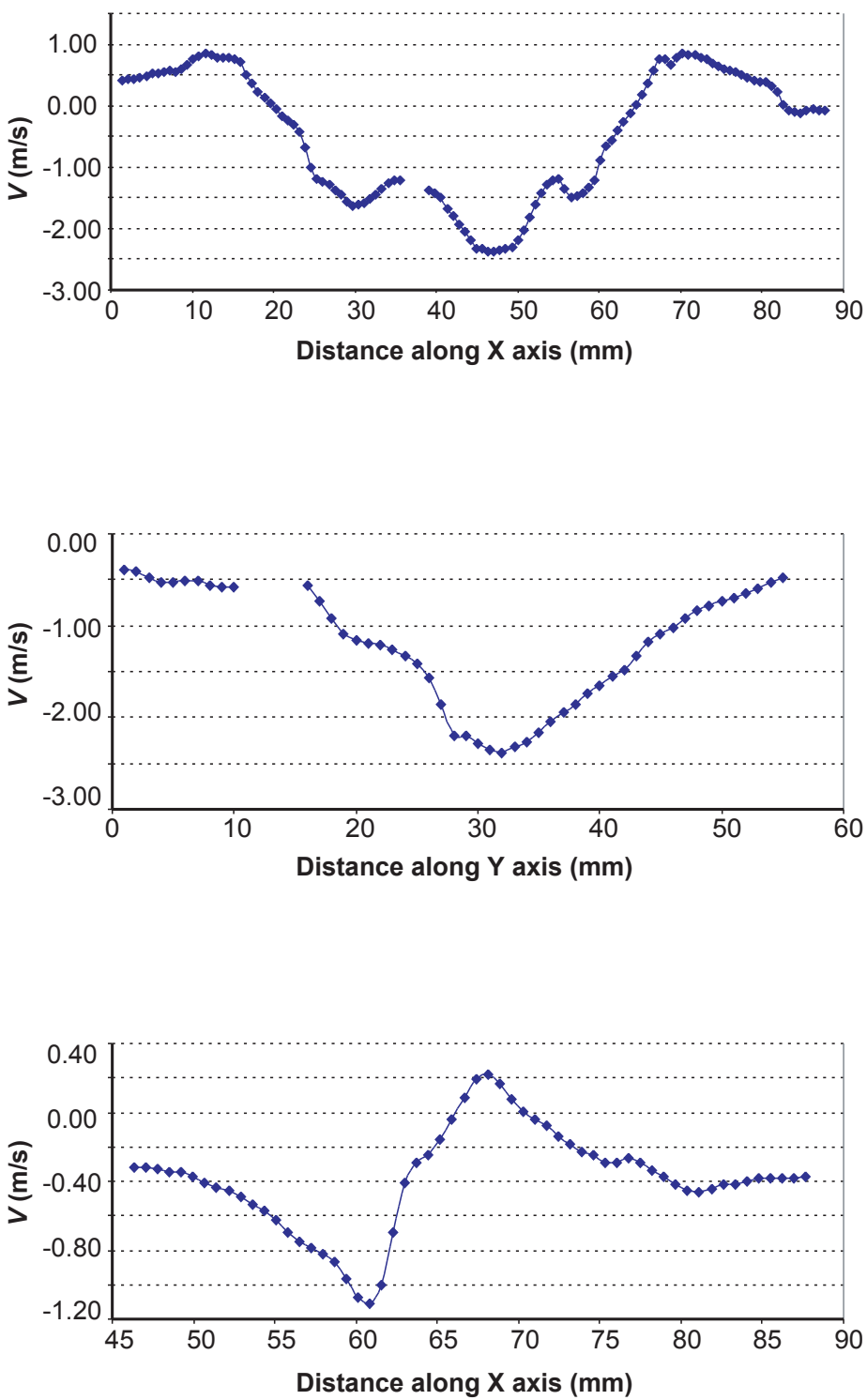

To show vertical and horizontal forces relative to body mass
Leading-Edge Vortex velocity profile
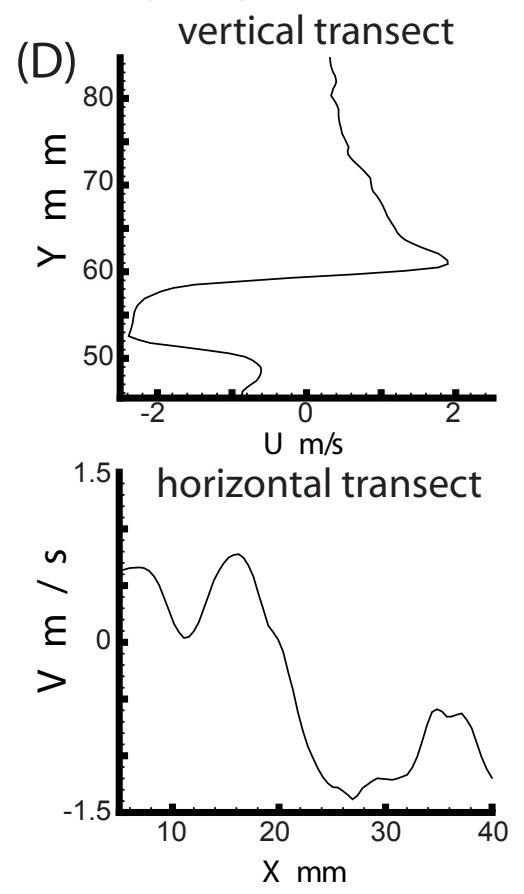

(E)

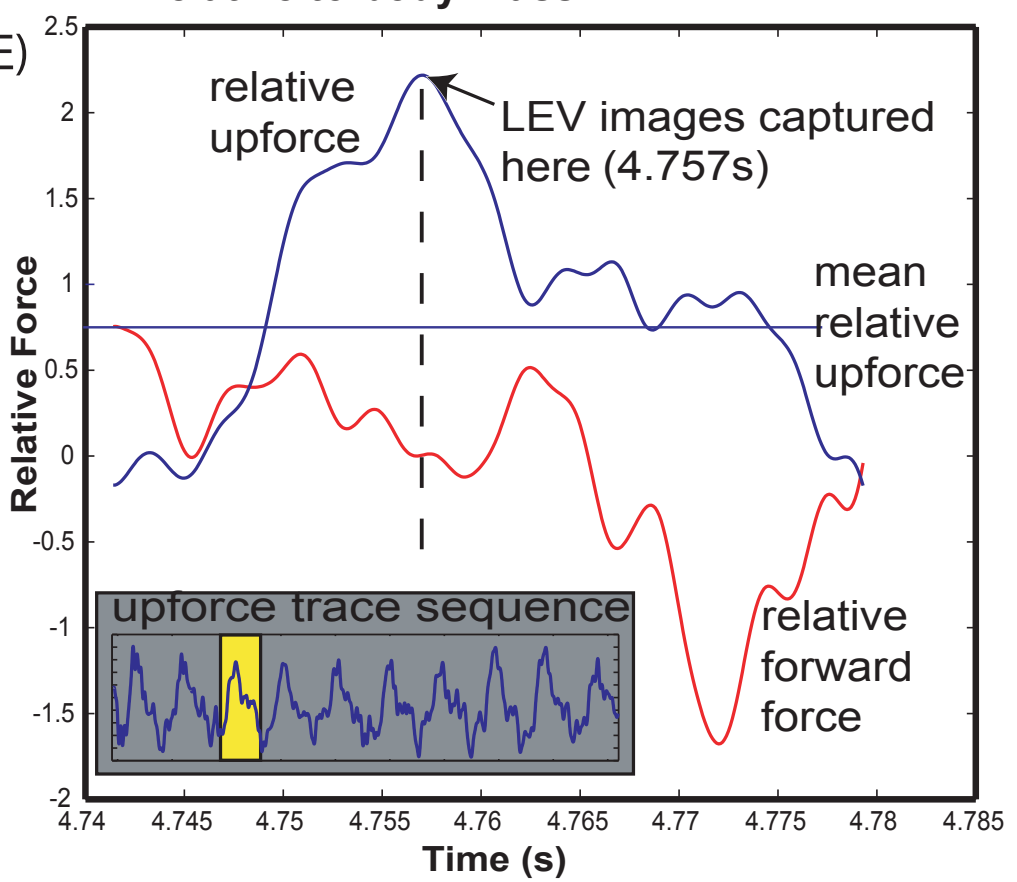



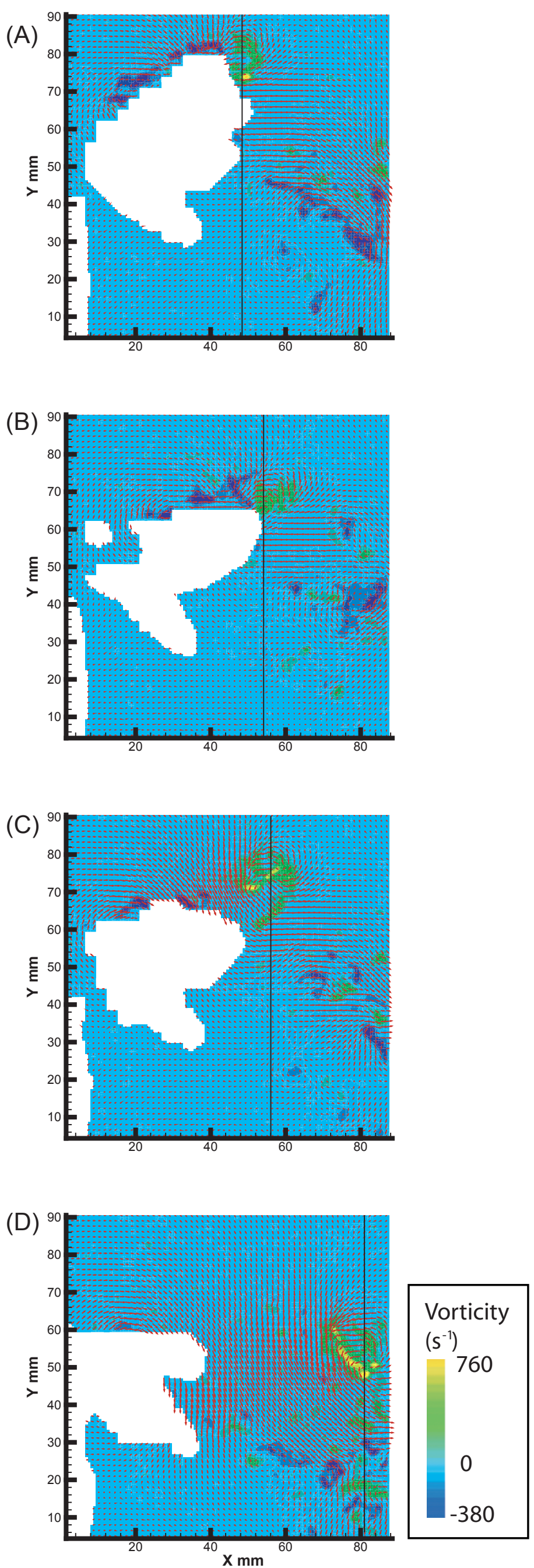

Bomphrey et al. Fig 4 

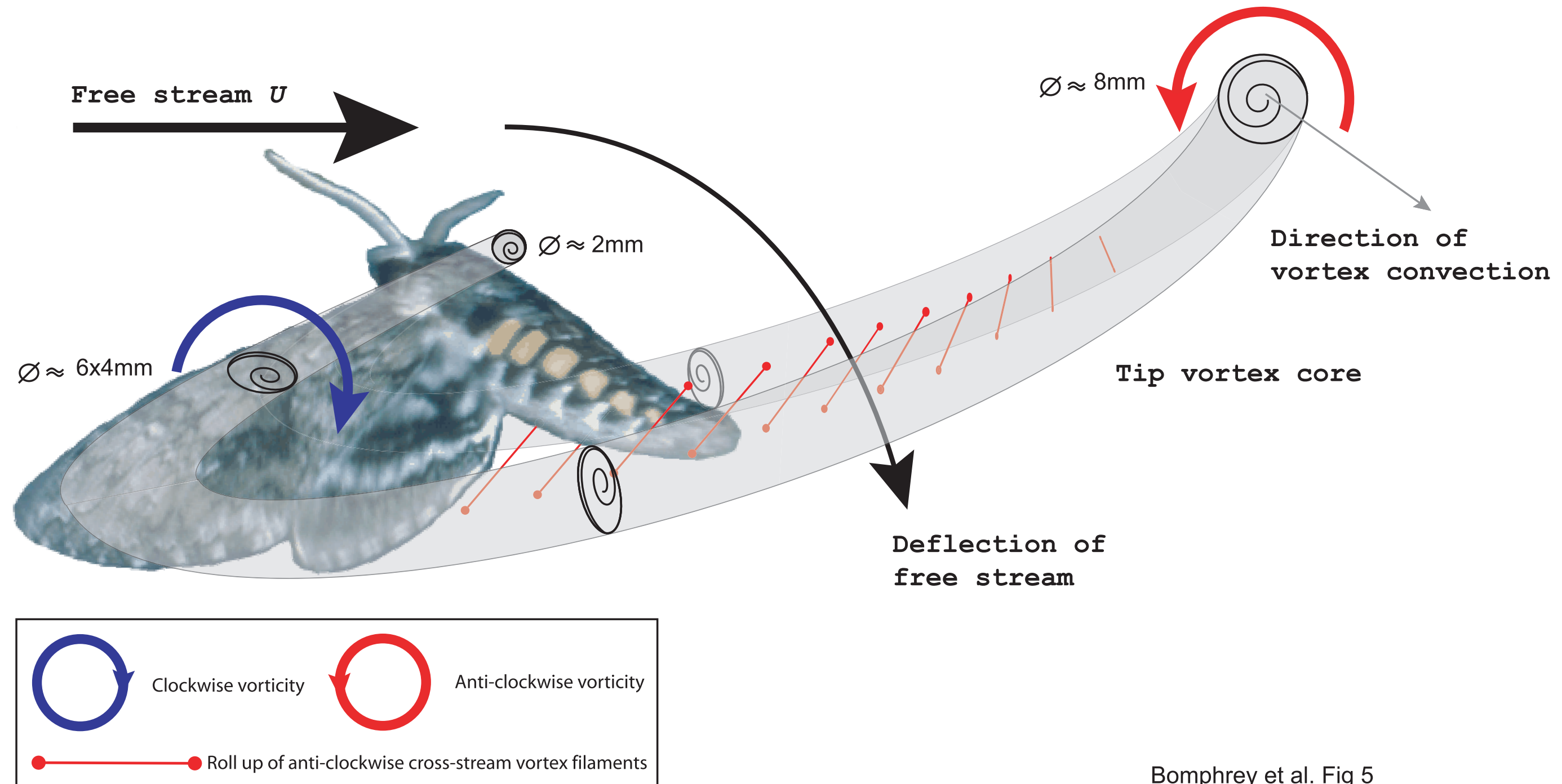

Bomphrey et al. Fig 5 
Bomphrey et al. Fig 6

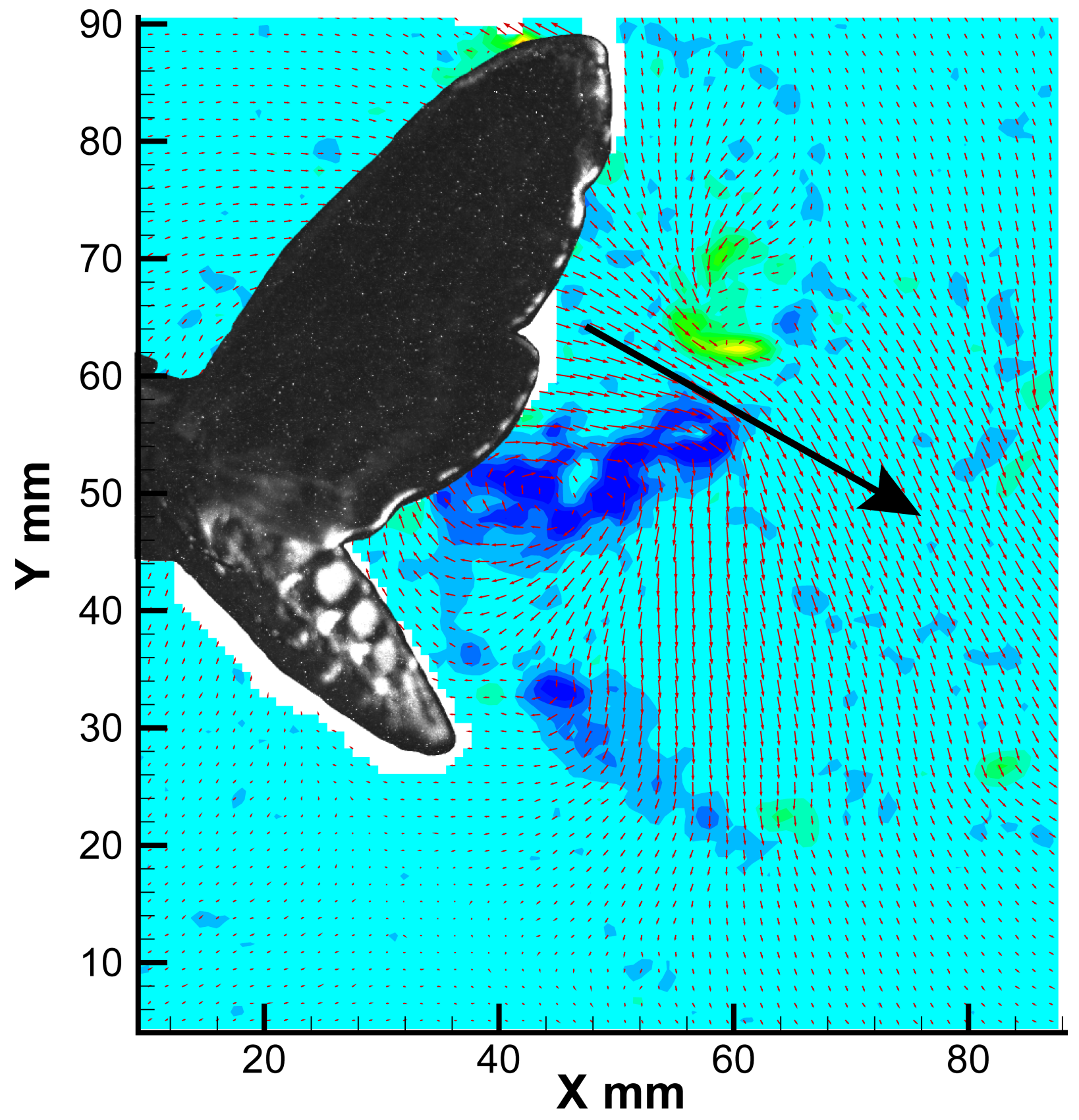

